University of Wollongong

Research Online

Australian Institute for Innovative Materials -

Papers

Australian Institute for Innovative Materials

$1-1-2016$

Effect of post-spinning on the electrical and electrochemical properties of wet spun graphene fibre

Javad Foroughi

University of Wollongong, foroughi@uow.edu.au

Dennis Antiohos

University of Wollongong, dennisa@uow.edu.au

Gordon G. Wallace

University of Wollongong, gwallace@uow.edu.au

Follow this and additional works at: https://ro.uow.edu.au/aiimpapers

Part of the Engineering Commons, and the Physical Sciences and Mathematics Commons

Research Online is the open access institutional repository for the University of Wollongong. For further information contact the UOW Library: research-pubs@uow.edu.au 


\title{
Effect of post-spinning on the electrical and electrochemical properties of wet spun graphene fibre
}

\author{
Abstract \\ There is an urgent need for electroactive materials that exhibit high electrochemical performance and \\ mechanically-compliant properties while also retaining high strength and durability. Single graphene \\ sheets have a large electroactive surface area and excellent electrical properties. However, to transfer the \\ excellent mechanical and physical properties of individual graphene to macrostructures for practical \\ application remains a challenge. Herein we demonstrate the effect of carbon nanotubes and/or \\ conducting polymer on electrical and electrochemical properties of graphene fibres. The hybrid graphene/ \\ carbon nanotube/poly(3,4-ethylenedioxythiophene) fiber possessed significantly higher electrical \\ conductivity (over $400 \mathrm{~S} \mathrm{~cm}-1$ ) compared to a graphene fiber. This value is $\approx 500 \%$ higher than the \\ reduced graphene oxide fiber. The graphene fibers show impressive electrochemical responses with a \\ specific capacitance in excess of $499 \mathrm{~F} \mathrm{~cm}-3$ at the scan rate of $5 \mathrm{mV} \mathrm{S}-1$. This was significantly \\ enhanced for hybrid graphene/CNT/PEDOT fiber by $\approx 221 \%$ compared to graphene fibre at the scan rate \\ of $1000 \mathrm{mV} \mathrm{s}-1$.

\section{Disciplines} \\ Engineering | Physical Sciences and Mathematics

\section{Publication Details} \\ Foroughi, J., Antiohos, D. \& Wallace, G. G. (2016). Effect of post-spinning on the electrical and \\ electrochemical properties of wet spun graphene fibre. RSC Advances, 6 (52), 46427-46432.
}




\title{
Effect of post-spinning on the electrical and electrochemical properties of wet spun graphene fibre
}

\author{
Javad Foroughi $^{\mathrm{a}^{*}}$, Dennis Antiohos ${ }^{\mathrm{a}}$, Gordon G. Wallace ${ }^{\mathrm{a}}$.
}

There is an urgent need for electroactive materials that exhibit high electrochemical performance and mechanicallycompliant properties while also retaining high strength and durability. Single graphene sheets have a large electroactive surface area and excellent electrical properties. However, to transfer the excellent mechanical and physical properties of individual graphene to macrostructures for practical application remains a challenge. Herein we demonstrate the effect of carbon nanotubes and/or conducting polymer on electrical and electrochemical properties of graphene fibres. The hybrid graphene/carbon nanotube /Poly(3,4ethylenedioxythiophene) fiber possessed significantly higher electrical conductivity (over $400 \mathrm{~S} \mathrm{~cm}^{-1}$ ) compared to a graphene fiber. This value is $\approx 500 \%$ higher than the reduced graphene oxide fiber. The graphene fibers show impressive electrochemical responses with a specific capacitance in excess of $499 \mathrm{~F} \mathrm{~cm}^{-3}$ at the scan rate of $5 \mathrm{mV} \mathrm{S}^{-1}$. This was significantly enhanced for hybrid graphene/CNT/PEDOT fiber by $\approx 221 \%$ compared to graphene fibre at the scan rate of $1000 \mathrm{mV} \mathrm{s}^{-1}$.

\section{Introduction}

Electronic textiles that incorporate functional elements such as energy storage and harvesting, sensors and actuators are limited by the difficulty in preparing textile fibres that combine excellent mechanical properties with needed multifunctionality. Graphene $(\mathrm{G})$ has recently attracted significant interest for use in areas including nanoelectronics, sensors, energy conversion and storage. ${ }^{[1-5]}$ To transfer the excellent mechanical and physical properties of individual graphene to macrostructures for practical application, numerous

${ }^{a}$ Intelligent Polymer Research Institute, ARC Centre of Excellence for Electromaterials Science, University of Wollongong, NSW, 2522, Australia efforts have been made to assemble graphene sheets into films or fibers. ${ }^{[6-11]}$ The range of properties available from such assemblies can be extended by incorporating other functional materials. ${ }^{[12-14]}$ Such materials can be used in areas that include advanced catalytic converters, battery electrodes and superconducting cables. ${ }^{[7,15-17]}$

The combination of graphene and their precursors with carbon nanotube (CNT) fibers led to the development of high performance electrodes for supercapacitors and batteries. ${ }^{[18-20]}$

In this paper we explore a new type of conductive nanocomposite fibers prepared by the post-spinning of a multi walled carbon nanotube (MWNT) and/or Poly(3,4-ethylenedioxythiophene) (PEDOT) on the surface of wet-spun graphene fibres. The procedure developed for the fabrication is substantially different from conventional composite fabrication processes in which graphene and carbon nanotube or conducting polymer fillers are mixed and then shaped to form a fiber or film. In our case, the liquid crystallite graphene oxide (LCGO) was formed into a fibre, then coated with the pre-formed MWNT sheets and/or PEDOT. The composite fibres exhibited improved electrical and electrochemical properties as compared with the 
pristine MWNT material, conducting polymer and graphene fibers. The composite fibers can be used for applications where the electrical conductivity along with excellent electrochemical properties are of primary importance.

\section{Experimental}

\subsection{Materials}

Ferric toluenesulfonate (Fe.pTS) in n-butanol (40\% v/v) (Clevios C-B40) was supplied by H.C. Starck and were used as received. EDOT $(3,4-$ ethylenedioxythiophene) monomer (95\%, Aldrich) was used after distillation. Graphite flakes was supplied by Asbury Graphite Mills USA.

\subsection{Wet spinning of Graphene fiber}

Graphene oxide (GO) is prepared from the intercalated graphite flakes. The liquid crystallite graphene oxide (LCGO) dispersion was prepared as described previously. ${ }^{[8]}$ Long, conductive, and flexible fibers were wet-spun from liquid crystallite graphene oxide (LCGO) dispersions where fiber coagulated with $\mathrm{CaCl}_{2}$ then reduced in vacuum at $220{ }^{\circ} \mathrm{C}$ as described previously. ${ }^{[8]}$

\subsection{Fabrication of hybrid graphene/CNT fiber}

The multi walled carbon nanotube forest was synthesized by catalytic CVD (chemical vapour deposition) using acetylene gas as the carbon source. Carbon nanotubes in the forest typically had a diameter of about $10 \mathrm{~nm}$ as described previously. ${ }^{[21,22]}$ The hybrid graphene MWNT fiber was prepared by post-spinning of wet-spun graphene fiber which MWNT fibers used for wrapping was directly drawn from a spinable MWNT forest around the surface of the asprepared graphene fiber. The MWNT fibers were wrapped around the graphene fiber layer by layer. After wrapping drops of ethanol were applied to densify the MWNT fibers and promote the interaction between the $\mathrm{CNT}$ and the graphene fibers. Finally, the hybrid graphene/CNT fibers were dried at $60{ }^{\circ} \mathrm{C}$ for $3 \mathrm{~h}$.

\subsection{Preparation of graphene composite fiber}

As-prepared graphene and graphene/CNT fibers were used to produce the graphene/PEDOT and graphene/CNT/PEDOT nanocomposite fibers. Graphene composite fibers were developed by chemical polymerization of EDOT through vapour phase polymerization. A length of as-prepared graphene and graphene/CNT fibers (Fig. 1 G, b1) were dipped into Fe.pTS (oxidant/dopant) solution (10\% v/v) for $10 \mathrm{~min}$ (Fig. $1 \mathrm{a} 1, \mathrm{~b} 2)$. The graphene and graphene/CNT coated with Fe.pTS were then dried at $60{ }^{\circ} \mathrm{C}$ for $15 \mathrm{~min}$. The dried graphene/Fe.pTS and graphene/CNT/Fe.pTS fibers were placed in a chamber containing monomer (EDOT) for $60 \mathrm{~min}$ at $75^{\circ} \mathrm{C}$. PEDOT then formed on and within the graphene and graphene/CNT fibers (Fig. 1 a2, b3). The graphene composites fibers were washed with methanol to remove salts and/ or monomer from the samples. As-prepared graphene/PEDOT and graphene/CNT/PEDOT fibres were dried at room temperature and used for further experiments. A comparison of the weight of sample before and after polymerization indicated 
that the weight fraction of the PEDOT in the graphene/PEDOT and graphene/CNT/PEDOT fibre $\left(\mathrm{cm}^{3}\right)$.

fibers were $\sim 5$ and $8 \mathrm{wt} \%$ respectively. ${ }^{[23-25]}$

\subsection{Characterization}

Electrochemical measurements (cyclic voltammetry and electrochemical impedance spectroscopy (EIS)) were performed using a Biologic VSP-300 instrument with EC-lab software V 10.37 using a two electrode set-up. The cyclic voltammetry potential window for aqueous $1 \mathrm{M}$ $\mathrm{H}_{2} \mathrm{SO}_{4}$ was between $0 \mathrm{~V}$ and $1 \mathrm{~V}$; while the potential window for $1 \mathrm{M} \mathrm{LiClO}_{4}$ in acetonitrile was between $0 \mathrm{~V}$ and $2 \mathrm{~V}$. The frequency range for EIS was between $100 \mathrm{kHz}$ and $0.1 \mathrm{~Hz}$ with a 10 $\mathrm{mV}$ (rms) perturbation voltage at open circuit potential. Series resistance calculations (Rs) were modelled using the equivalent circuit modelling packaging on EC-lab V 10.37.

Samples were prepared by integrating the asprepared fibers into fine micron sized Pt wires in order to provide a good current collection. The graphene fiber and its composites were then laminated onto a PET sheet in order to provide mechanical support. $\mathrm{Cu}$ tape was then pressed onto the end of the fine Pt wire so electrical connection could be achieved to the potentiostat. The capacitance $(\mathrm{C})$ is calculated by Equation 1 . I is the response current density (A) calculated from the integral of the current with respect to time (i.e. charge), obtained from cyclic voltammetry. The limits of the integral are the potential window (V), and $\alpha$ is either the mass ( $\mathrm{g}$ ) of one electrode, the area of one electrode $\left(\mathrm{cm}^{2}\right)$ or the volume of one

$$
C=\frac{1}{V \alpha} \int I d t
$$

The morphology of as-prepared graphene fibers were characterized by a JEOL JSM-7500 FESEM. Samples for imaging were prepared by cutting cross-sections in liquid nitrogen using a scalpel blade. They were then coated with a thin $(10 \mathrm{~nm})$ layer of platinum $(\mathrm{Pt})$ to aid with imaging and minimize beam heating effects. Cross-sections were analysed at $25 \mathrm{kV}$ accelerating voltage and a spot size setting of 13 under high vacuum. Electrical transport of the graphene fiber and hybrid graphene fibers was characterized by a standard four probe technique (Quantum Design PPMS). All measured fibers were placed onto $\mathrm{MgO}$ substrate and four $\mathrm{Au}$ wires were attached sample using silver paste. The dependence of conductivity $(\sigma)$ on temperature $(\mathrm{T})$ was measured by sweeping the temperature from $350 \mathrm{~K}$ to $5 \mathrm{~K}$ with a sweep rate of $3 \mathrm{~K} / \mathrm{min}$. 


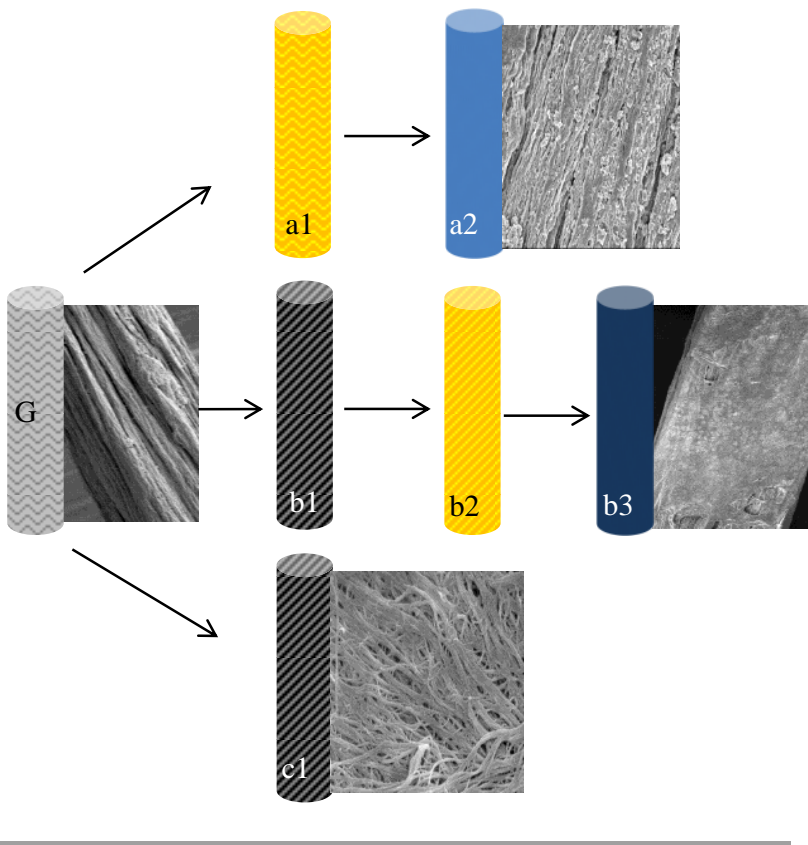

Fig.1 Schematic diagram to illustrate the hybrid graphene fiber process. (b1, c1) wrapping spinable MWNT sheet around a graphene fiber, (a1, b2) coating graphene and graphene/CNT fibers with Fe.PTS (oxidant/dopant), and (a2, b3) polymerisation of EDOT onto surface of fibres.

\section{Results and discussion}

The wet spun graphene fiber and its composites can be produced continuously without limitation in length. Hybrid graphene fibers were formed using the processes illustrated schematically in Figure 1. MWNTs were wrapped around as-prepared graphene fiber to fabricate the graphene/CNT fiber. To form the graphene conducting polymer fiber either graphene or the graphene/CNT fibers were passed through the oxidant/dopant bath. Fibers were dried and the monomer (EDOT) introduced into the fibers to form PEDOT.

\subsection{Morphology of as-prepared graphene fibres}

SEM micrographs of the wet spun graphene fiber and its composite are shown in Fig. 2. As can be seen from the surface morphology and crosssection, the graphene sheets were uniform and predominantly oriented to the fiber axis (Fig. 2 a1a3). The as-prepared hybrid graphene/PEDOT fiber was slightly rough and non-uniform coating of PEDOT (Fig.2 b1-b3). Some areas of the fiber displayed a surface morphology similar to that of the pristine graphene fiber, suggesting little incorporation of PEDOT (Fig. 2 b2). SEM micrographs of the graphene/CNT fibers showed a core-sheath structure with a graphene inner core and a CNT sheath (Fig. 2 c1-c3). The sheath thickness was 1-1.5 $\mu \mathrm{m}$ (Fig. $2 \mathrm{c} 3$ ) and the nanotubes were uniform and mainly oriented with a helix angle $(\alpha)$ of $\sim 45^{\circ}$ to the fiber axis. The asprepared graphene/CNT/PEDOT nanocomposite fiber also showed a core-sheath structure with a graphene inner core and a CNT/PEDOT sheath (Fig. 2 d1-d3). SEM micrographs of the graphene/CNT/PEDOT fiber shows a uniform coating of PEDOT and the surface was less porous which CNTs were only visible at higher magnifications. 


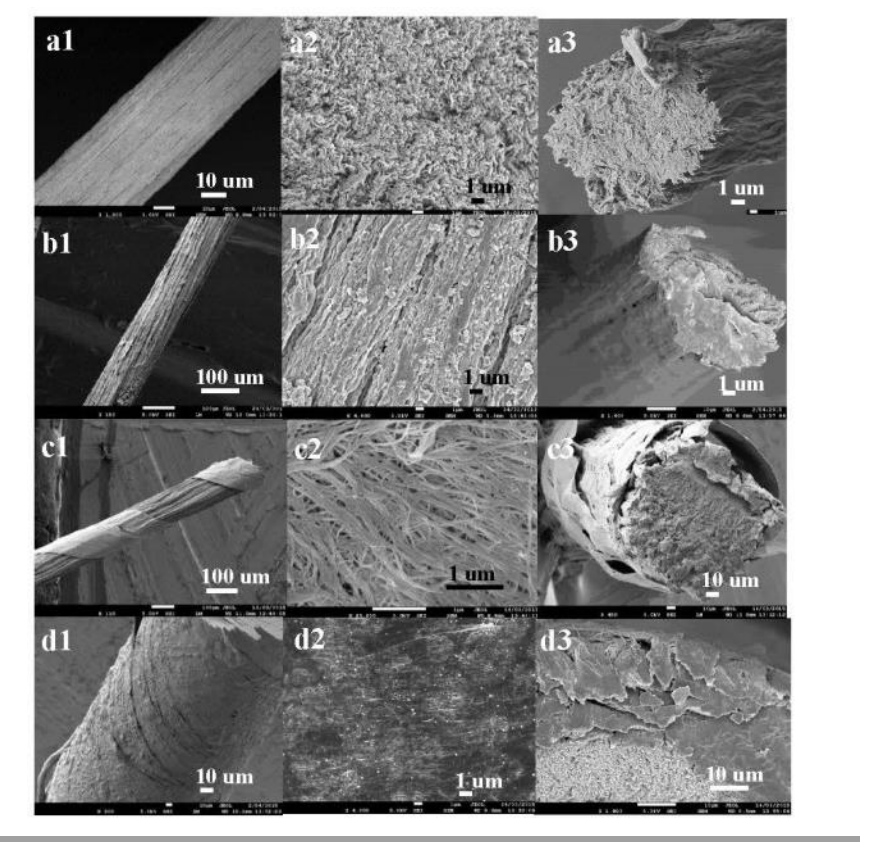

Figure 2: SEM micrographs of wet spun graphene fiber at (a1) the surface morphology, (a2a3) fiber in cross-section at low and higher magnification; SEM micrographs of graphene/PEDOT fiber showing the surface morphology of a fiber at (b1) low and (b2) higher magnification and (b3) fiber in cross-section; SEM micrographs of graphene/CNT fiber showing the surface morphology at (c1) low, (c2) higher magnification and (c3) fiber in cross-section. Image (d1-d3) show the surface morphology of the graphene/CNT/PEDOT fiber at low, (d2) higher magnification and (d3) cross-sectional

\subsection{Electrical properties of as-prepared graphene fiber}

Figure 3 compares the temperature dependence of the conductivity, for the fibres. As can be seen from Fig. 3, PEDOT enhances significantly the conductivity of graphene/CNT fiber (over 441 $\mathrm{S} / \mathrm{cm})$. This value is $147 \%, 507 \%$, and $735 \%$ higher than CNT yarn, graphene fiber and PEDOT naonfiber, respectively. ${ }^{[8,21,22,26]}$ It is expected that PEDOT acts as a binder in the graphene/CNT fiber and therefore reduces the slippage between CNT bundles and improves the charge carrier mobiltiy.
As a result, the electrical conductivity of the fiber is enhanced due to better connection between CNTs. The conductivity of samples at room temperature was 87, 130, 176 and $441 \mathrm{~S} / \mathrm{cm}$, for graphene, graphene/PEDOT, graphene/CNT and graphene/CNT/PEDOT fibers, respectively.The conductivity of all samples decreased linearly with decreasing temperature down to $5 \mathrm{~K}$.

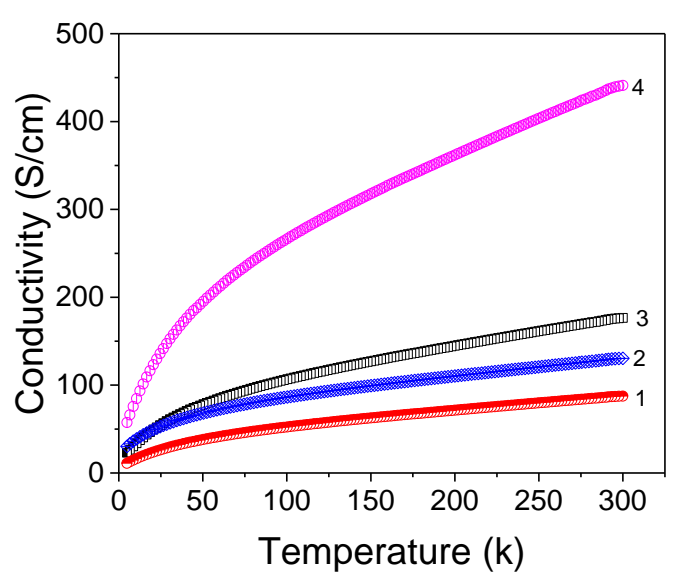

Figure 3: the temperature dependence of electrical conductivity of the as-prepared (1) graphene fiber, (2) graphene/PEDOT, (3) graphene/CNT and (4) graphene/CNT/PEDOT composite fibers.

\subsection{Electrochemical properties of as-prepared graphene fiber \\ 3.3.1 Performance in aqueous electrolyte}

The general trend of the of the Nyquist plot (Fig. 4a) shows that at low frequency, the imaginary part of the impedance ( $\operatorname{Im~Z)~approaches~a~general~}$ vertical behaviour indicating capacitance where there is finite diffusion of ions as the boundary comes into play. ${ }^{[27]}$ In the high-middle frequency portion of the spectrum, semi-circles are observed indicating a charge transfer resistance as ions are migrating during the charging / discharging process 
at the electrode /electrolyte interface. ${ }^{[28,29]}$ The largest semi-circle is for the graphene fiber $(\approx 35$ Ohm. $\mathrm{cm}^{2}$ ). (see Table 1) In the CV comparison of figure $4 \mathrm{~b}$, the largest currents are observed for Graphene/CNT/PEDOT, Graphene/PEDOT, and Graphene. Using just Graphene/CNT did not produce a significant current due to wettability issues with the aqueous electrolyte. In Table 1 the capacitance was calculated using equation 1 and it can be seen that the Graphene and Graphene/CNT/PEDOT had the largest specific capacitances as a function of mass, area and volume. The specific capacitance was enhanced by $205 \%$ for the fabricated graphene/CNT/PEDOT fiber compared to graphene fiber at high scan rate $(1000 \mathrm{mV} / \mathrm{s})$ due to higher electrical conductivity of developed graphene/CNT/PEDOT nanocomposite fibers.

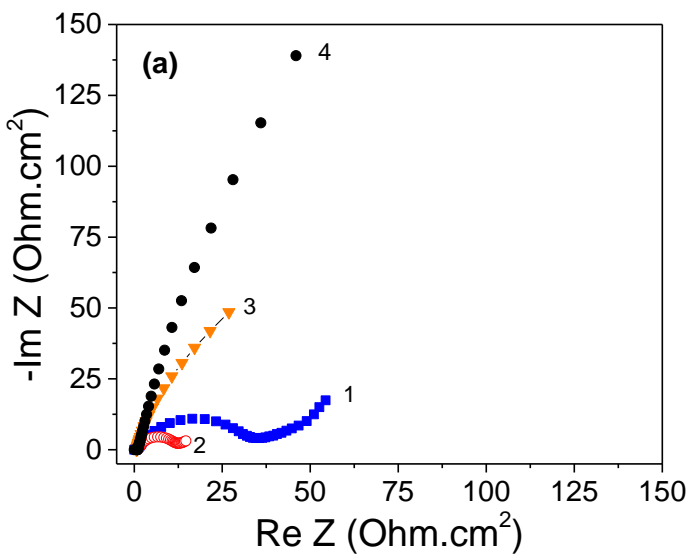

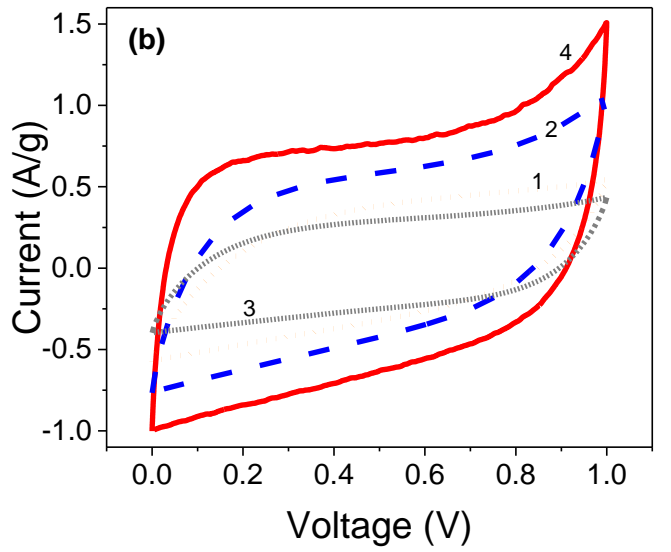

Figure 4: (a) Nyquist plot and (b) $\mathrm{CV}$ at $1 \mathrm{~V} / \mathrm{s}$ of different composite fibers 1) graphene, 2) graphene/PEDOT, 3) graphene/CNT and 4) graphene/CNT/PEDOT. Electrolyte is $1 \mathrm{M} \mathrm{H}_{2} \mathrm{SO}_{4}$.

\subsubsection{Performance in organic electrolyte}

The general trend of the Nyquist plot (Fig. 5a) shows that in the low frequency portion of the spectrum, the imaginary impedance $(\operatorname{Im} \mathrm{Z})$ approaches a horizontal line indicating partially resistive behaviour and infinite diffusion as the boundary is far enough away and does not come into play. ${ }^{[27]}$ In the high-middle frequency portion of the spectrum, semi-circles are observed, once again indicating a charge transfer resistance as ions are migrating during the charging / discharging process at the electrode / electrolyte interface. ${ }^{[28,29]}$ The largest semi-circle is for the graphene fiber $(\approx$ 20 Ohm. $\mathrm{cm}^{2}$ ). (see Table 1) The charge transfer resistance of the graphene fiber in $1 \mathrm{M} \mathrm{LiClO}_{4} /$ acetonitrile is approximately $40 \%$ less than that in 1 $\mathrm{M} \mathrm{H}_{2} \mathrm{SO}_{4} / \mathrm{H}_{2} \mathrm{O}$ meaning that there is a higher affinity for the organic electrolyte with the graphene as the wettability is enhanced. In the CV 
comparison of Figure 5b, the largest currents are observed for Graphene/CNT and Graphene. The composite fibers with PEDOT had a lower current density due to wettability issues as acetonitrile does not wet PEDOT very well. In Table 1 the capacitance was calculated at the scan rate of 5 $\mathrm{mV} / \mathrm{s}$ using equation 1 and it can be seen that the Graphene had the largest specific capacitances as a function of mass, area and volume at $106 \mathrm{~F} / \mathrm{g}, 0.93$ $\mathrm{F} / \mathrm{cm}^{2}$, and $373 \mathrm{~F} / \mathrm{cm}^{3}$ respectively. As a comparison, in previous work Foroughi et. al. reported MWNT-graphene yarns with a specific capacitance of $111 \mathrm{~F} / \mathrm{g}$ in $1 \mathrm{M} \mathrm{LiClO}_{4}$ / acetonitrile. ${ }^{[18]}$ Mirfakhrai et al. reported pristine CNT yarns that achieved a specific capacitance of $\begin{array}{lllll}26 & \mathrm{~F} / \mathrm{g} & \text { in } & 0.5 & \mathrm{M}\end{array}$ tetrabutylammoniumtetrafluoroborate/acetonitrile.

${ }^{[30]}$ Lee et al. reported a volumetric capacitance 179 $\mathrm{Fcm}^{-3}\left(1 \mathrm{M} \mathrm{H}_{2} \mathrm{SO}_{4}\right)$ for PEDOT/MWNT yarns that were wrapped around a Pt wire for electrochemical testing. ${ }^{[31]}$ As result of higher electrical conductivity developed graphene/CNT/PEDOT fiber, its specific capacitance at scan rate of 1000 $\mathrm{mV} / \mathrm{s}$ was $221 \%$ higher than graphene fiber.
Table 1: Capacitance and series resistance for as-prepared graphene fibres

\begin{tabular}{|c|c|c|c|c|c|c|}
\hline Sample & $\begin{array}{l}\text { Capaci } \\
\text { @ } 5 \mathrm{~m}\end{array}$ & ce $\left(\mathrm{F} / \mathrm{cm}^{3}\right)$ & $\begin{array}{l}\text { Capaci } \\
\left(\mathrm{F} / \mathrm{cm}^{3}\right. \\
@ 100\end{array}$ & $\begin{array}{l}\text { ance } \\
\mathrm{mV} / \mathrm{s}\end{array}$ & $\begin{array}{l}\text { Rs } \\
\text { (Ohm.c }\end{array}$ & \\
\hline Electrolyte & $\mathrm{H}_{2} \mathrm{SO}_{4}$ & $\mathrm{LiClO}_{4}$ & $\mathrm{H}_{2} \mathrm{SO}_{4}$ & $\mathrm{LiClO}_{4}$ & $\mathrm{H}_{2} \mathrm{SO}_{4}$ & $\mathrm{LiClO}_{4}$ \\
\hline $\begin{array}{l}\text { Graphene/CNT/PED } \\
\text { OT }\end{array}$ & 490.7 & 208.5 & 28.5 & 19.6 & 0.9 & 3.4 \\
\hline Graphene/CNT & 33.1 & 271.2 & 2.4 & 8.1 & 0.6 & 8.1 \\
\hline Graphene/ PEDOT & 222.0 & 99.2 & 20.6 & 1.5 & 1.1 & 3.8 \\
\hline Graphene & 499.1 & 373.5 & 14.0 & 8.9 & 0.8 & 3.5 \\
\hline
\end{tabular}
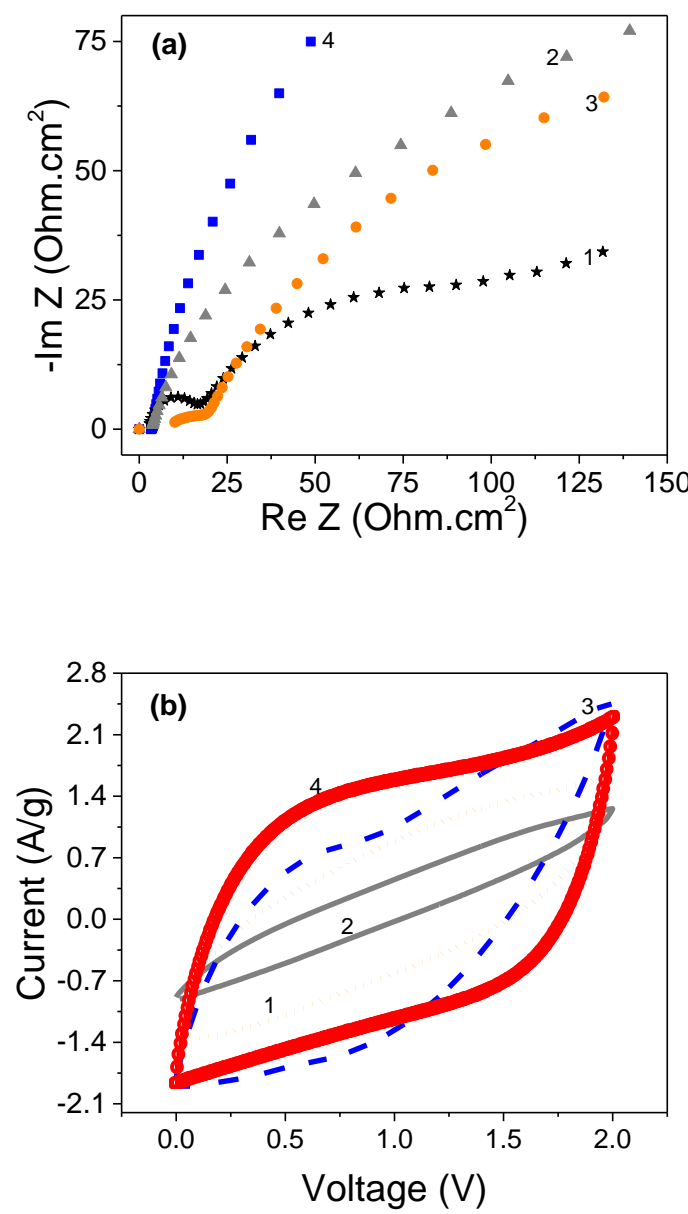

Figure 5: (a) Nyquist plot and (b) $\mathrm{CV}$ at $1 \mathrm{~V} / \mathrm{s}$ of different composite fibers 1) graphene, 2) graphene/PEDOT, 3) graphene/CNT and 4) graphene/CNT/PEDOT. Electrolyte is $1 \mathrm{M} \mathrm{LiClO}_{4}$.

\section{Conclusion}

We explore architecture of nanostructured electroactive materials with a view to enhance their electrical and electrochemical properties. Highly 
conductive graphene nanocomposite fibers were fabricated through a continuous and simple post spinning method. Four types of wet spun graphene and graphene composite fibers were developed and compared in terms of electrical and electrochemical properties. Due to the excellent interfacial bonding between the CNT, graphene and PEDOT, the electrical conductivity of developed structure can be significantly enhanced. The results suggest that the electrical conductivity of the hybrid graphene/CNT/PEDOT fiber is over $441 \mathrm{~S} / \mathrm{cm}$. This value is 5 orders magnitude higher than graphene fiber. The areal specific capacitance reaches $490.71 \mathrm{~F} / \mathrm{cm}^{3}$ and $499.05 \mathrm{~F} / \mathrm{cm}^{3}$ for graphene /CNT/PEDOT and graphene fibres, respectively at the scan rate of $5 \mathrm{mVs}^{-1}$. The specific capacitance enhanced by $221 \%$ for graphene /CNT/PEDOT fiber compared to graphene fiber while the scan rate increased to $1000 \mathrm{mVs}^{-1}$ due to its higher electrical conductivity. Such substantial improvements of key properties of the hybrid material can be associated with the synergy of CNT, PEDOT and graphene layers in the fiber structure. Prepared hybrid fibers can benefit as high-performance soft, flexible electrodes for biomedical applications such neural stimulation and recording electrodes.

\section{Acknowledgements}

J. Foroughi thanks the ARC under Discovery Early Career Researcher Award (DE12010517). The authors also thank the ANFF Materials Node for their provision of research facilities, the Global
Challenges Seed Funding and acknowledge the use of facilities within UOW Electron Microscopy Centre. The authors would like to thank Professor Ray Baughman (University of Texas at Dallas) for his continuous support, insights and also for providing the CNT forests needed for producing the fibers.

\section{References}

1. Fang, B., et al., Wet-Spinning of Continuous Montmorillonite-Graphene Fibers for FireResistant Lightweight Conductors. ACS Nano, 2015. 9(5): p. 5214-5222.

2. $\mathrm{Xu}, \mathrm{Z}$. and C. Gao, Graphene in Macroscopic Order: Liquid Crystals and Wet-Spun Fibers. Accounts of Chemical Research, 2014. 47(4): p. 1267-1276.

3. Kou, L., et al., Coaxial wet-spun yarn supercapacitors for high-energy density and safe wearable electronics. Nat Commun, 2014. 5.

4. Liao, Q., et al., All-Solid-State Symmetric Supercapacitor Based on Co3O4 Nanoparticles on Vertically Aligned Graphene. ACS Nano, 2015. 9(5): p. 5310-5317.

5. Zhao, X., et al., Graphene-based single fiber supercapacitor with a coaxial structure. Nanoscale, 2015. 7(21): p. 9399-9404.

6. Chen, D., H. Feng, and J. Li, Graphene oxide: preparation, functionalization, and electrochemical applications. Chemical reviews, 2012. 112(11): p. 6027-6053.

7. Dikin, D.A., et al., Preparation and characterization of graphene oxide paper. Nature, 2007. 448(7152): p. 457-460.

8. Jalili, R., et al., Scalable One - Step Wet Spinning of Graphene Fibers and Yarns from Liquid Crystalline Dispersions of Graphene Oxide: Towards Multifunctional Textiles. Advanced Functional Materials, 2013. 23(43): p. 5345-5354.

9. Foroughi, J., et al., Torsional Carbon Nanotube Artificial Muscles. Science, 2011. 334(6055): p. 494-497.

10. Xiang, C., et al., Large flake graphene oxide fibers with unconventional 100\% knot efficiency 
and highly aligned small flake graphene oxide fibers. Advanced Materials, 2013. 25(33): $p$. 4592-4597.

11. $\mathrm{Xu}, \mathrm{Z}$., et al., Ultrastrong fibers assembled from giant graphene oxide sheets. Advanced Materials, 2013. 25(2): p. 188-193.

12. Wang, H., et al., Graphene oxide doped polyaniline for supercapacitors.

Electrochemistry Communications, 2009. 11(6): p. 1158-1161.

13. Williams, G., B. Seger, and P.V. Kamat, TiO2graphene nanocomposites. UV-assisted photocatalytic reduction of graphene oxide. ACS nano, 2008. 2(7): p. 1487-1491.

14. Zhu, X., et al., Nanostructured reduced graphene oxide/Fe2O3 composite as a highperformance anode material for lithium ion batteries. Acs Nano, 2011. 5(4): p. 3333-3338.

15. Becerril, H.A., et al., Evaluation of solutionprocessed reduced graphene oxide films as transparent conductors. ACS nano, 2008. 2(3): p. $463-470$.

16. Gómez-Navarro, C., et al., Electronic transport properties of individual chemically reduced graphene oxide sheets. Nano letters, 2007. 7(11): p. 3499-3503.

17. Apollo, N.V., et al., Soft, Flexible Freestanding Neural Stimulation and Recording Electrodes Fabricated from Reduced Graphene Oxide. Advanced Functional Materials, 2015: p. n/an/a.

18. Foroughi, J., et al., Highly Conductive Carbon Nanotube-Graphene Hybrid Yarn. Advanced Functional Materials, 2014. 24(37): p. 58595865.

19. Kholmanov, I.N., et al., Optical, Electrical, and Electromechanical Properties of Hybrid Graphene/Carbon Nanotube Films. Advanced Materials, 2015. 27(19): p. 3053-3059.

20. Shin, M.K., et al., Synergistic toughening of composite fibres by self-alignment of reduced graphene oxide and carbon nanotubes. Nat Commun, 2012. 3: p. 650.

21. Zhang, M., K.R. Atkinson, and R.H. Baughman, Multifunctional Carbon Nanotube Yarns by Downsizing an Ancient Technology. Science, 2004. 306(5700): p. 1358-1361.

22. Zhang, M., et al., Strong, Transparent, Multifunctional, Carbon Nanotube Sheets. Science, 2005. 309(5738): p. 1215-1219.
23. Foroughi, J., et al., Preparation and characterization of hybrid conducting polymercarbon nanotube yarn. Nanoscale, 2012. 4(3): p. 940-945.

24. Kim, J.Y., et al., Self-Assembly and Crystalline Growth of Poly(3,4-ethylenedioxythiophene) Nanofilms. Advanced Materials, 2007. 19(21): p. 3501-3506.

25. Winther-Jensen, B., et al., High Rates of Oxygen Reduction over a Vapor Phase-Polymerized PEDOT Electrode. Science, 2008. 321(5889): $\mathrm{p}$. 671-674.

26. Laforgue, A. and L. Robitaille, Production of conductive PEDOT nanofibers by the combination of electrospinning and vaporphase polymerization. Macromolecules, 2010. 43(9): p. 4194-4200.

27. Bard, A.J. and L.R. Faulkner, Fundamentals and applications. Electrochemical Methods, 2nd ed.; Wiley: New York, 2001.

28. Bose, S., et al., Carbon-based nanostructured materials and their composites as supercapacitor electrodes. Journal of Materials Chemistry, 2012. 22(3): p. 767-784.

29. Rakhi, R., et al., High performance supercapacitors using metal oxide anchored graphene nanosheet electrodes. Journal of Materials Chemistry, 2011. 21(40): p. 1619716204.

30. Tissaphern, M., et al., Electrochemical actuation of carbon nanotube yarns. Smart Materials and Structures, 2007. 16(2): p. S243.

31. Lee, J.A., et al., Ultrafast charge and discharge biscrolled yarn supercapacitors for textiles and microdevices. Nature communications, 2013. 4. 